\title{
Secant Dimensions of Minimal Orbits: Computations and Conjectures
}

\author{
Karin Baur, Jan Draisma, and Willem A. de Graaf
}

\section{CONTENTS}

1. Introduction

2. The Algorithm

3. Implementation

4. Conjectures

Acknowledgments

References

2000 AMS Subject Classification: Primary 14N05; Secondary 14Q15, $14 \mathrm{~L} 35$

Keywords: Projective techniques, higher-dimensional varieties, classical groups
We present an algorithm for computing the dimensions of higher secant varieties of minimal orbits. Experiments with this algorithm lead to many conjectures on secant dimensions, especially of Grassmannians and Segre products. For these two classes of minimal orbits we give a short proof of the relation-known from the work of Ehrenborg, Catalisano-Geramita-Gimigliano, and Sturmfels-Sullivant-between the existence of certain codes and nondefectiveness of certain higher secant varieties.

\section{INTRODUCTION}

A generic polynomial of degree $d$ in $\mathbb{C}[x]$ can be written as a sum of $\lfloor(d+1) / 2\rfloor$ powers $(a x+b)^{d}, a, b \in \mathbb{C}$. A generic $n \times n$ matrix of rank $k$ and trace 0 is the sum of $k$ matrices of rank 1 and trace 0 ; in fact, this is true for any tracezero matrix of rank $k$, though that doesn't matter here. But what is the generic rank of a tensor in $\left(\mathbb{C}^{2}\right)^{\otimes 10}$ ? That is, if we want to write a generic element of this tensor power as a sum of decomposable tensors, then how many do we need?

These are instances of a general type of problem that has been solved only in very few cases. In this paper, we do not solve many instances either, but we do present a program for investigating small concrete instances. Also, we will boldly state some conjectures that our experiments with this program suggest. We hope that this paper will be an incentive for others working in this field either to prove or disprove our conjectures, or to use our program and experiment for themselves.

To be more concrete about the type of problem that our program can handle, let $G$ be a connected reductive complex algebraic group and let $V$ be a nontrivial irreducible module for $G$; for the theory of algebraic groups we refer to [Borel 91]. The projective space $\mathbb{P} V$ contains a unique (Zariski-)closed orbit $X$, consisting of the highestweight lines and called the minimal orbit; see Section 2 for the short argument. Denote by $C \subseteq V$ the affine cone 
over $X$. For any natural number $k$, we write $k X$ for the Zariski closure of the union of all projective $(k-1)$-spaces spanned by $k$ points on $X ; k X$ is called the $k$ th secant variety of $X$ (by some other authors the $(k-1)$ st secant variety). Often, the term secant variety itself is used for the second secant variety, while those for $k>2$ are referred to as higher secant varieties. More concretely, the affine cone over $k X$ is the Zariski closure of

$$
k C:=\left\{v_{1}+\cdots+v_{k} \mid v_{i} \in C\right\} .
$$

In the examples above, $G$ is equal to $\mathrm{SL}_{2}$ acting on the space $V$ of binary forms of degree $d$, or to $\mathrm{SL}_{n}$ acting on its Lie algebra, or to $\mathrm{SL}_{2}^{10}$ acting on the space $\left(\mathbb{C}^{2}\right)^{\otimes 10}$ of 10-tensors, respectively. Accordingly, $C$ is the set of pure $d$ th powers of linear forms, or the set of trace-zero matrices of rank at most 1 , or the set of all pure 10tensors.

One can ask many questions about the sets $k X$ and $k C$. For instance, how do we find polynomial equations defining $\overline{k C}$ ? For the matrix example we know them: $k C=\overline{k C}$ is the set of trace-zero matrices of rank at most $k$, and these are characterized (even scheme-theoretically; see [Bruns and Vetter 88]) by the vanishing of all $(k+1)$ minors. For the example of binary forms, too, equations defining $\overline{k C}$ set-theoretically are known: certain minors of Hankel (or catalecticant) matrices [Harris 92, Proposition 9.7] (indeed, these equations even define $\overline{k C}$ schemetheoretically; see, e.g., [Iarrobino and Kanev 99]).

But for the example of 10-tensors we do not know equations. Of course, equations can in theory be found by a Gröbner basis computation: One computes the closure of the image of the addition morphism $C^{k} \rightarrow k C$. However, these computations quickly become totally infeasible, so sophisticated algebraic geometry is already needed to find equations for small secant varieties. There is recent progress in this direction: In [Landsberg and Weyman 06], scheme-theoretic equations for some secant varieties of 3-fold and 4-fold Segre powers are given, and [Sturmfels and Sullivant 06] shows how combinatorial methods help in finding such equations.

Another question: Are the sets $k C$ closed? In the matrix case they are; for the 10-tensors we do not know; and for the binary forms they are not. One can show, in fact, that a polynomial with a zero of multiplicity $m$, $0<m<d$, cannot be written as a sum of fewer than $m+1$ pure $d$ th powers, so that the sets $k C$ with $\lfloor(d+$ 1) $/ 2\rfloor \leq k<d$ cannot possibly be closed.

But by far the most modest property of $k C$ that one may want to determine is its dimension $\operatorname{dim} k C:=$ $\operatorname{dim} \overline{k C}=\operatorname{dim} k X+1$, and this is precisely what our algorithm does. That such an algorithm is useful is clear from the vast literature dealing exactly with these secant dimensions; see, e.g., [Alexander 88, Alexander and Hirschowitz 92, Alexander and Hirschowitz 95, Catalisano et al. 02, Catalisano et al. 05a, Catalisano et al. 05b, Ehrenborg 00, Hirschowitz 85, Sturmfels and Sullivant 06].

Since the addition map $C^{k} \rightarrow \overline{k C}$ is dominant, $\operatorname{dim} k C$ is at most $k \operatorname{dim} C$; we call the minimum of the latter number and $\operatorname{dim} V$ the expected dimension of $k C$. If $k C$ has the expected dimension, then $k C$ (and $\overline{k C}$ and $k X$ ) are called nondefective. Otherwise, $k C, \overline{k C}$, and $k X$ are called defective. The difference $\min \{\operatorname{dim} V, k \operatorname{dim} C\}-$ $\operatorname{dim} k C$ is called the $k$-defect (note that these defects are the sums of the defects as defined in [Zak 93]). If $k C$ is not defective for any $k \geq 1$, then we call $C$ and $X$ themselves nondefective; otherwise, we call them defective.

As we will see below, calculating $\operatorname{dim} k C$ in concrete cases boils down to straightforward linear algebra computations - at least if one allows for a small error probability - and only in rare concrete cases does $k C$ not have the expected dimension. And yet it is very difficult to prove anything substantial in this direction.

First, however, we list some important things that are known about these higher secant varieties. The standard reference for secant varieties, containing a wealth of classification results on varieties with constraints on their secant dimensions, is [Zak 93]:

1. Take $G=\mathrm{SL}_{n}$ and let $V$ be the space of homogeneous polynomials in $x_{1}, \ldots, x_{n}$ of degree $d$. Then $C$ is the set of $d$ th powers of linear forms. A simple duality shows that $\operatorname{dim} k C$ is the codimension of the space of homogeneous polynomials in $x_{1}, \ldots, x_{n}$ of degree $d$ that vanish together with all their first partial derivatives on $k$ fixed generic points. This relates higher secant varieties to the problem of multivariate interpolation, which was solved in the series of papers [Hirschowitz 85, Alexander 88, Alexander and Hirschowitz 92, Alexander and Hirschowitz 95].

2. For $G$ a simple algebraic group acting on its Lie algebra $\mathfrak{g}$, the set $C$ consists of all "extremal elements," that is, elements $X \in \mathfrak{g}$ for which $\operatorname{ad}(X)^{2} \mathfrak{g} \subseteq \mathbb{C} X$. The first secant variety is known in this case ([Kaji et al. 99, Kaji and Yasukura 00]), and for classical $G$ the higher secant varieties were completely determined in [Baur and Draisma 04]. 
3. For $G=\mathrm{SL}_{n}$ and $V$ the $d$-fold exterior power of $\mathbb{C}^{n}$, the set $C$ is the affine cone over the Grassmannian, in its Plücker embedding, of $d$-dimensional vector spaces in $\mathbb{C}^{n}$. The paper [Catalisano et al. 05a] lists some defective Grassmannians, and proves that for $d>2$ and $k d \leq n$ the variety $k C$ is not defective. In Section 4.1 we generalize this latter result, and conjecture that the list of defective Grassmannians in [Catalisano et al. 05a] is complete.

4. For $G=\mathrm{SL}_{n_{1}} \times \cdots \times \mathrm{SL}_{n_{d}}$ and $V=\mathbb{C}^{n_{1}} \otimes \cdots \otimes \mathbb{C}^{n_{d}}$, the cone $C$ consists of the decomposable powers, and is the cone over the Segre product of the $\mathbb{C}^{n_{i}}$. For small $d$ and some concrete values of the $n_{i}$, secant dimensions of $C$, or bounds on these, are known; see [Catalisano et al. 02] and the references therein. The case in which all $n_{i}$ are equal is treated in more detail in Section 4.

From here we proceed as follows: in Section 2 we present our algorithm for computing $\operatorname{dim} k C$; Section 3 deals with some implementation issues; and Section 4 lists our conjectures based on experiments with that implementation. These conjectures concern Grassmannians and Segre products, as well as a general finiteness statement. Our algorithm, called sedimo, can be downloaded from http://www.win.tue.nl/ jdraisma/.

\section{THE ALGORITHM}

We retain the notation $G, V, X, C$ from Section 1 . That is, $G$ is a connected reductive algebraic group, $V$ is a nontrivial irreducible $G$-module, $X$ is the closed orbit in $\mathbb{P} V$-whose uniqueness will be dealt with soon - and $C$ is the cone over $X$ in $V$.

\subsection{Required Representation Theory}

For those whose background is not in representation theory, we briefly sketch how one can put the objects under investigation into a computer algebra system to compute secant dimensions: Let $B$ be a Borel subgroup of $G$, i.e., maximal under the closed, connected subgroups of $G$, and let $T$ be a maximal torus in $B$. Then $B$ stabilizes a unique 1-dimensional subspace in $V$, spanned by a highest-weight vector $v_{0}$, and the character of $T$ on this line determines $V$ up to isomorphism. This character is the highest weight and can be given by a tuple of natural numbers relative to a certain basis of the (free abelian) character group of $T$. Conversely, algorithms are already available in [GAP 02] to construct, given a highest weight as a tuple of natural numbers, the corresponding module
$V$-strictly speaking not as a $G$-module, but as a module over the Lie algebra $\mathfrak{g}$ of $G$. The tangent space to the orbit $G v_{0}$ in $V$ is $\mathfrak{g} v_{0}$, and transporting this space around by elements of $G$ constructed with the exponential map $\mathfrak{g} \rightarrow G$ gives tangent spaces that are used in the computation of $\operatorname{dim} k C$; eventually, the rank of some large matrix is our lower bound on $\operatorname{dim} k C$. All these matters can be found in [Borel 91, Humphreys 81, Humphreys 71].

Unfortunately, the computations are rather slow in characteristic 0, and therefore we indicate in Section 3 how to reduce them modulo a prime. Very roughly speaking, one first does the construction above over $\mathbb{Z}$; i.e., one constructs elements of $G$ that have integral matrices relative to some appropriate basis of $V$. Then the large matrix whose rank needs to be computed is also integral, and hence its rank modulo $p$ is a lower bound to its rank over $\mathbb{Q}$. The insight that these constructions can in theory be done over $\mathbb{Z}$ (and hence over any field) goes back to Chevalley [Steinberg 68]. The results on quantum groups that the actual implementation in Section 3 uses cannot be given extensive description in the present paper.

\subsection{The Highest-Weight Orbit}

We reduce the computation of $\operatorname{dim} k C$ to straightforward linear algebra as follows. First, we recall that the closed $G$-orbit in $\mathbb{P} V$ is unique and equal to the orbit of highest-weight lines: Indeed, suppose that $x \in \mathbb{P} V$ has a closed orbit $G x$, and let $P$ be the stabilizer of $x$ in $G$. Then $G / P \cong G x$ is a projective variety, and therefore $P$ contains a Borel subgroup $B$ of $G$ [Borel 91, Section 11.2, Corollary]. By the representation theory of algebraic groups and their Lie algebras [Borel 91, Humphreys 71, Humphreys 81], B stabilizes a unique line in the irreducible representation $V$, namely, the highest-weight line $\mathbb{C} v_{0}$, which therefore corresponds to $x$. Hence $X=G x$ is the orbit of highest-weight lines. Then the cone $C$ over the minimal orbit $X$ equals $C=G v_{0} \cup\{0\}$-recall that $V$ was assumed nontrivial, so that this really is a cone. In what follows we need the Lie algebras $\mathfrak{g}, \mathfrak{p}$ of $G$ and $P$, and also a maximal torus $T$ of $B$.

\subsection{A Dense Orbit under a Unipotent Subgroup}

Now let $\mathfrak{u}_{-}$be the direct sum of all $T$-root spaces in $\mathfrak{g}$ that are not in $\mathfrak{p}$. Then $\mathfrak{u}_{-}$is the Lie algebra of a unique connected (unipotent) subgroup $U_{-}$of $G$. Let $X_{1}, \ldots, X_{r}$ be a basis of $\mathfrak{u}_{-}$consisting of $T$-root vectors. Then the following statements are well known: 
1. The map $\Psi: \mathbb{C}^{r} \rightarrow U_{-}$sending $\left(t_{1}, \ldots, t_{r}\right)$ to $\exp \left(t_{1} X_{1}\right) \cdots \exp \left(t_{r} X_{r}\right)$ is an isomorphism of varieties (combine Section 14.4, Proposition, with Section 7.3 from [Borel 91]).

2. The $U_{-}$-orbit $U_{-} v_{0}$ is the intersection of $C$ with the affine hyperplane where the $v_{0}$-coordinate is 1 (relative to a $T$-weight basis of $V$ containing $v_{0}$ ). This follows easily from the Bruhat decomposition [Borel 91, Section 14.12, Theorem].

3. Hence the image of $U_{-} v_{0}$ in $\mathbb{P} V$ is dense in $X$.

Our program works, in fact, with elements in $U_{-} v_{0}$ rather than all of $C$.

\subsection{Terracini's Lemma}

We work here with $\tilde{C}:=G v_{0}=C \backslash\{0\}$, which is a smooth variety since it is homogeneous. Consider the addition $\operatorname{map} \pi: \tilde{C}^{k} \rightarrow \overline{k C},\left(v_{1}, \ldots, v_{k}\right) \mapsto \sum_{i} v_{i}$ and denote its differential at the $k$-tuple $\mathbf{v}=\left(v_{1}, \ldots, v_{k}\right) \in \tilde{C}^{k}$ by $d_{v} \pi$. By elementary algebraic geometry, the map sending $\mathbf{v} \in \tilde{C}^{k}$ to the rank of $d_{\mathbf{v}} \pi$ is lower semicontinuous (since $\tilde{C}^{k}$ is smooth), and its generic value is $\operatorname{dim}(k C)$ by the dominance of $\pi$. On the other hand, the image of $d_{\mathbf{v}} \pi$ equals

$$
\sum_{i=1}^{k} T_{v_{i}} \tilde{C}
$$

where $T_{v_{i}} \tilde{C}$ denotes the tangent space to $C$ at $v_{i}$, regarded as a linear subspace of $V$. We conclude that the dimension of this latter space is always a lower bound for $\operatorname{dim} k C$, while it is equal to $\operatorname{dim} k C$ for generic tuples $\mathbf{v}$. This observation is, in fact, one of the first results in the theory of join and secant varieties, and is due to Terracini [Terracini 11].

\subsection{The Algorithm}

Our algorithm, which we have implemented in GAP [GAP 02], is as follows:

\section{Algorithm 2.1.}

Input: $(\mathfrak{g}, \lambda, k)$, where $\mathfrak{g}$ is a split rational semisimple Lie algebra with a distinguished split Cartan subalgebra $\mathfrak{h}$ and a distinguished Borel subalgebra $\mathfrak{b}$ containing $\mathfrak{h} ; \lambda \in \mathfrak{h}^{*}$ is a $\mathfrak{b}$-dominant weight; and $k$ is a natural number.

Output: a lower bound for $\operatorname{dim} k C$ that with high probability equals $\operatorname{dim} k C$. (Here $C=G v_{0} \cup\{0\}$, where $G$ is the simply connected complex algebraic group associated to $\mathfrak{g}$ and $v_{0}$ is a $\mathfrak{b}$-highest-weight vector in the irreducible $\mathfrak{g}$ - (and $G$-) module of highest weight $\lambda$.)

\section{Method:}

1. Construct the irreducible representation $\rho$ : $\mathfrak{g} \rightarrow \mathfrak{g l}(V)$ of highest weight $\lambda$.

2. Denote by $v_{0}$ the highest-weight vector of $V$, and compute representatives $\left(X_{1}, \ldots, X_{r}\right)$ of the negative $\mathfrak{h}$-root spaces in $\mathfrak{g}$ that do not vanish on $v_{0}$ (these span $\left.\mathfrak{u}_{-}\right)$.

3. For $i \in\{1, \ldots, r\}$ compute those divided powers $\rho\left(X_{i}\right)^{d} /(d !)$ that are nonzero.

4. Set $T:=\{0\}$, the zero subspace of $V$.

5. Compute $T_{v_{0}} C:=K v_{0}+\mathfrak{u}_{-} v_{0}$.

6. Repeat the following steps $k$ times:

(a) Choose rational numbers $t_{1}, \ldots, t_{r}$ at random.

(b) Compute

$$
u:=\exp \left(t_{1} \rho\left(X_{1}\right)\right) \cdots \exp \left(t_{r} \rho\left(X_{r}\right)\right)
$$

using the divided powers of the $\rho\left(X_{i}\right)$ for faster computation of the exponentials.

(c) Set $T:=T+u T_{v_{0}} C$.

7. Return $\operatorname{dim} T$.

Proof of the algorithm: The isomorphism $\Psi$ of Section 2.3 identifies $U_{-}$as an affine space, and moreover gives $U_{-}$a $\mathbb{Q}$-structure, corresponding to $\mathbb{Q}^{r}$ via $\Psi$. In the $i$ th step of the algorithm, a random element $u_{i}$ of $U_{-}(\mathbb{Q})$ is computed, as well as the corresponding tangent space $T_{u_{i} v_{0}} C=u_{i} T_{v_{0}} C$. The algorithm returns the dimension of the sum of these $T_{u_{i} v_{0}} C$. We claim that this dimension is smaller than $\operatorname{dim} k C$ only if $\left(u_{1}, \ldots, u_{k}\right)$ lies in some proper Zariski-closed subset of $U_{-}(\mathbb{Q})^{k}$ - hence only with "small probability." Indeed, since $T_{s v} C=T_{v} C$ for all $s \in \mathbb{C}^{*}$ and $v \in U_{-} v_{0}$, and since $\left\{s v \mid s \in \mathbb{C}^{*}, v \in\right.$ $\left.U_{-} v_{0}\right\}$ is dense in $C$, the generic rank of $d_{\mathbf{v}} \pi$ for $\mathbf{v} \in C^{k}$ is equal to the generic rank of $d_{\mathbf{v}} \pi$ for $\mathbf{v} \in\left(U_{-} v_{0}\right)^{k}$. Hence the $\left(u_{1}, \ldots, u_{k}\right) \in U_{-}^{k}$ where $d_{\left(u_{1} v_{0}, \ldots, u_{k} v_{0}\right)} \pi$ does not have rank $\operatorname{dim} k C$ form a proper closed subset $S$ of $U_{-}^{k}$. Finally, $U_{-}(\mathbb{Q})^{k}$ is Zariski-dense in $U_{-}^{k}$, so that it cannot be contained in $S$. This proves the correctness of the algorithm. 
It is hard to estimate, in an implementation of this algorithm, the precise probability with which the selected tuple $\left(u_{1}, \ldots, u_{k}\right)$ lies outside $S$, since it depends on the random generator supplying the rational numbers $t_{i}$. However, if the algorithm returns the expected dimension, then we are sure that the output is correct. Otherwise, by repeating the procedure, we can make the probability that the result is correct arbitrarily close to 1 .

\section{IMPLEMENTATION}

We have implemented the algorithm in the computer algebra system GAP4 [GAP 02], using the built-in functionality for semisimple Lie algebras and their representations. All steps are rather straightforward to implement. It turns out that in working over the field $\mathbb{Q}$, the main bottleneck of the algorithm is the computation of a basis of the space $T$ in Step 6(c). For example, the computation of the secant dimensions of the Grassmannian of 5-dimensional subspaces in an 11-dimensional vector space took 362 seconds, of which 309 were spent in the basis computation of Step 6(c). ${ }^{1}$ This is due to the fact that the coefficients of the vectors grow very fast (probably because of their random nature). In the example mentioned earlier, the vectors in a triangularized basis were dense, and contained rational numbers of up to 70 digits in both denominator and numerator.

For this reason we have included in our implementation the possibility to compute modulo a prime $p$. This, however, presents a new problem: The coefficients of the matrices of the divided power in Step (3) may not be integral. We can get around this by computing an "admissible lattice" in the highest-weight module $V$ (cf. [Humphreys 71]). An algorithm for this purpose is not present in GAP4. We have implemented an algorithm for this based on the theory of crystal bases (cf. [Jantzen $96])$.

Roughly, this works as follows. First we note that $V$ is also a module for the quantum group $U_{q}(\mathfrak{g})$. Now from the crystal graph of $V$ we get a set of elements $F_{i}$ in the negative part of $U_{q}(\mathfrak{g})$, with the property that $\left\{F_{i} \cdot v_{0}\right\}$ spans an admissible lattice (for details we refer to [de Graaf 03, Lakshmibai 95]).

Each $F_{i}$ can be mapped to an element $F_{i}^{\prime}$ of the negative part of the universal enveloping algebra $U(\mathfrak{g})$. Then $\left\{F_{i}^{\prime} \cdot v_{0}\right\}$ spans an admissible lattice of $V$. This approach has the advantage that we do not need to check linear

\footnotetext{
${ }^{1}$ The computations in this section were done on a $2-\mathrm{GHz}$ processor, with 500 MB RAM memory for GAP.
}

\begin{tabular}{|r|r|r|r|r|r|r|}
\hline$n$ & $d$ & Total & Module & Basis & $\operatorname{dim} V(\lambda)$ & $k_{\max }$ \\
\hline 11 & 5 & 71 & 60 & 3 & 462 & 14 \\
12 & 6 & 222 & 165 & 23 & 924 & 24 \\
13 & 6 & 973 & 686 & 160 & 1716 & 39 \\
14 & 7 & 5456 & 3249 & 1380 & 3432 & 68 \\
\hline
\end{tabular}

TABLE 1. Time (in seconds) for the computation of the secant dimensions of the Grassmannian of $d$-spaces in $n$-space. The third column lists the total time spent; the fourth and fifth, respectively, the time used for the construction of the module along with the matrices in Step (3), and for the computation of the basis in Step 6(c). The sixth column displays the dimension of the $\mathfrak{g}$-module, and the last column contains $k_{\max }$, the maximal $k$ for which $\operatorname{dim} k C$ has been computed.

independence of the basis elements. The necessary algorithms for quantum groups are implemented in the GAP4 package QuaGroup [de Graaf 05].

With this, the computation in the example above took 71 seconds, with only 3 seconds spent in Step 6(c).

In computing modulo a prime, the computed dimensions may be smaller than those over $\mathbb{Q}$. However, we have an upper bound for the dimension of $k C$ (namely $k \operatorname{dim} C$ ), which "usually" gives the correct dimension. Furthermore, if this bound is attained by our algorithm, then we are sure to have the correct value. It rarely happens that this upper bound is not reached. However, if this happens to be the case, then we perform the computation modulo a bigger prime, and eventually over $\mathbb{Q}$. If we still do not attain the upper bound in that case, we conclude that we are in a defective situation with high probability.

Another problem occurs when the dimension of $V$ gets large (e.g., close to 1000). Then storing the matrices in Step (3) may lead to memory problems. To get around this we used an ad hoc implementation of sparse matrices (storing only the nonzero entries). This greatly reduces the memory requirements, and for dimensions greater than roughly 500 leads to a speedup for the matrix multiplications in Step 6 (b) as well.

Table 1 contains some run times of the algorithm in computing the secant dimensions of the Grassmannian of $d$-dimensional subspaces of $n$-space. We see that the running times increase rather sharply, mainly because the same holds for the dimensions of the $\mathfrak{g}$-modules. Most of the time is spent on the construction of the module $V(\lambda)$ and the matrices of Step (3). For small $n$, the time used in Step 6(c) is negligible, but as $n$ increases, the percentage of the time spent in that step also increases. 


\section{CONJECTURES}

\subsection{Grassmannians}

For $G=\mathrm{SL}_{n}$ and $V=\bigwedge^{d}\left(\mathbb{C}^{n}\right)$, the minimal orbit $X$ is the Grassmannian, in its Plücker embedding, of $d$ dimensional vector subspaces of $\mathbb{C}^{n}$, and the cone $C$ over $X$ is the set of (completely) decomposable wedge products in $V$. For this setting, our algorithm more or less reduces to the algorithm presented in [McGillivray 05], in which article the following conjecture is also tested up to $n=14$. For $d=2$ the set $k C$ equals is the set of all skew-symmetric matrices of (usual matrix) rank at most $2 k$ (see, e.g., [Zak 93] or [Baur and Draisma 04]); we therefore exclude $d=2$ in the following conjecture.

Conjecture 4.1. Suppose that $d>2$ and also that $2 d \leq n$. Then $C$ is defective in exactly the following cases:

1. $n=7$ and $d=3$, in which case $(\operatorname{dim} k C)_{k}$ equals $(13,26, * 34,35)$;

2. $n=8$ and $d=4$, in which case $(\operatorname{dim} k C)_{k}$ equals $(17,34, * 50, * 64,70)$; or

3. $n=9$ and $d=3$, in which case $(\operatorname{dim} k C)_{k}$ equals $(19,38,57, * 74,84)$,

where $*$ indicates the defective dimensions.

We have verified this conjecture with our program for all $n$ up to 15 . The defective Grassmannians in the list above were already found in [Catalisano et al. 05a]. For each of them, the first defect occurs at the smallest $k$ with $k d>n$. The following proposition shows that there are no other defective Grassmannians with this property.

Proposition 4.2. In the setting of Conjecture 4.1, write $n=q d+r$ with $0 \leq r<d$. Then $k C$ is not defective for $k=1, \ldots, q$, and $(q+1) C$ is defective if and only if $(n, d, q+1) \in\{(7,3,3),(8,4,3),(9,3,4)\}$.

The statement for $k \leq q$ is already present in [Catalisano et al. 05a].

Proof: For $k \leq q+1$ the group $\mathrm{SL}_{n}$ has a dense orbit on the $k$-fold Cartesian power $X^{k}$. Indeed, choose a basis

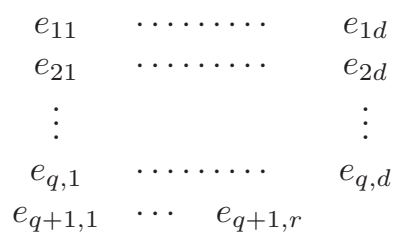

of $\mathbb{C}^{n}$. For every $d$-subset $S$ of

$$
\begin{gathered}
A:=\{(i, j) \mid 1 \leq i \leq q+1,1 \leq j \leq d, \\
\text { and } i=q+1 \Rightarrow j \leq r\},
\end{gathered}
$$

we let $e_{S}$ denote the wedge product (say in normal reading order) of the $e_{i j}$ with $(i, j) \in S$; these form a basis of $V=\bigwedge^{d} \mathbb{C}^{n}$. For $i=1, \ldots, q$ let $v_{i}$ be the wedge product of the $i$ th row in the array above. For $j=r+1, \ldots, d$ let $s_{j}$ be the sum of the $d$ th column in the array above, and set

$$
v_{q+1}:=e_{q+1,1} \cdots e_{q+1, r} s_{r+1} \cdots s_{d} .
$$

One readily verifies that the $\mathrm{GL}_{n}$-orbit of the tuple $\left(\mathbb{C} v_{i}\right)_{i=1}^{k}$ is dense in $X^{k}$ for all $k=1, \ldots, q+1$. Therefore, the generic rank of the summation map $C^{k} \rightarrow k C$ equals its rank at the tuple $\left(v_{1}, \ldots, v_{k}\right)$. In other words, the dimension of $k C$ is equal to the dimension of the sum

$$
T_{v_{1}} C+\cdots+T_{v_{k}} C .
$$

Now for $i \leq q$, the tangent space $T_{v_{i}} C$ is the span of all $e_{S}$ where $S$ is a $d$-subset of $A$ containing at least $d-1$ elements from row $i$. Using this description, the assumption $d>2$ readily implies that the sum

$$
T:=T_{v_{1}} C+\cdots+T_{v_{q}} C
$$

is direct. This proves that $\operatorname{dim}(k C)=k \operatorname{dim}(C)$ for $k \leq q$.

Now suppose that $k=q+1$. Then the above shows that $k C$ is defective if and only if $T_{v_{k}}$ contains a nonzero element of $T$. An element of $T_{v_{k}}$ is of the form

$$
\begin{aligned}
w:= & \sum_{j=1}^{r} e_{k, 1} \cdots b_{j} \cdots e_{k, r} s_{r+1} \cdots s_{d} \\
& +\sum_{j=r+1}^{d} e_{k, 1} \cdots e_{k, r} s_{r+1} \cdots b_{j} \cdots s_{d},
\end{aligned}
$$

where the $b_{j} \in \mathbb{C}^{n}$ replaces $e_{k, j}$ in the first sum and $s_{j}$ in the second sum. Let $S$ be a $d$-set in $A$; we will compute the coefficient of $e_{S}$ in $w$. First, $e_{S}$ can occur in $w$ only if $S$ is of one of the following four types:

Type 1. $S$ contains the entire last ( $k$ th) row of $A$, and furthermore $S$ hits all $d-r$ last columns of $A$.

Type 2. $S$ is obtained from a Type- 1 set by replacing a single position in the $k$ th row, say $(k, j)$, with $j \leq r$, by position $(i, j)$ with $i<k$ in the same column.

Type 3. $S$ is obtained from a Type- 1 set by replacing a single position in the $k$ th row, say $(k, j)$, by position $\left(i, j^{\prime}\right)$, with $i<k$ and $j^{\prime} \neq j$, i.e., in a different column. 
Type 4. $S$ is obtained from a Type-1 set by replacing a single position in the last $d-r$ columns, say $(i, j)$, with $i<k$ and $j>r$, with a position $\left(i^{\prime}, j^{\prime}\right)$ with $i^{\prime}<k$ and $j^{\prime} \neq j$, i.e., in a different column.

For computing the coefficient of $e_{S}$ in $w$ in these four cases, we let $B: A \rightarrow \mathbb{C}$ be the function mapping $(i, j)$ to the $(i, j)$ th component of $b_{j}$; note that $B$ records only the entries of $b_{j}$ corresponding to basis elements of $\mathbb{C}^{n}$ in column $j$. Then the coefficients are as follows:

1. If $S$ is of Type 1 , then $e_{S}$ can occur in all terms of $w$ (and, in fact, also in $v_{k}$ ), and its coefficient in $w$ is $\pm \sum_{(i, j) \in S} B(i, j)$ (the sign is irrelevant).

2. If $S$ is of Type 2 , then $e_{S}$ can occur only in the term of $w$ with index $j$, and its coefficient is $\pm B(i, j)$.

3. If $S$ is of Type 3 or 4 , then $e_{S}$ can occur only in the term of $w$ with index $j$, and there is a unique column of $A$ containing two elements of $S$, say $\left(i_{1}, j^{\prime}\right),\left(i_{2}, j^{\prime}\right)$ with $i_{1}<i_{2}$ and $j^{\prime} \neq j$. Now there are two cases:

(a) If $j^{\prime} \leq r$, then $i_{2}$ is equal to $k=q+1$, and $e_{S}$ has coefficient $\pm\left(b_{j}\right)_{\left(i_{1}, j^{\prime}\right)}$ (which is not recorded by $B !)$.

(b) If $j^{\prime}>r$, then the coefficient of $e_{S}$ is $\pm\left(\left(b_{j}\right)_{\left(i_{1}, j^{\prime}\right)}-\left(b_{j}\right)_{\left(i_{2}, j^{\prime}\right)}\right)$ : One chooses either $e_{i_{1}, j^{\prime}}$ as the $j$ th factor and $e_{i_{2}, j^{\prime}}$ as the factor at position $j^{\prime}$, or vice versa. Again, these coefficients are not in $B$.

Now suppose that $w \in T$. Then the only $e_{S}$ that can have nonzero coefficient in $w$ are those for which $S$ is obtained from one of the first $q$ rows of $A$ by replacing a single entry by another entry of $A$. Such $d$-sets $S$ we will call good, others bad. For every bad $S$ of Types $1-4$, the above yields a linear condition on the coefficients of the $b_{j}$ reflecting that $e_{S}$ has coefficient 0 in $w$. We will first argue that we may assume the coefficients $\left(b_{j}\right)_{\left(i, j^{\prime}\right)}$ with $j^{\prime} \neq j$, i.e., those not recorded by $B$, to be zero.

By assumption, $d \geq 3$ and $n \geq 2 d \geq 6$, so $q \geq 2$. The case $n=6=2 d$ needs special attention: Note that then $T_{v_{1}} C \oplus T_{v_{2}} C=V$, so that $C$ is not defective. So we assume that either $d \geq 4$ or $n>2 d$. Then it is somewhat tedious, but straightforward, to verify that the linear conditions for $S$ of Types 3 and 4 show that for all $j=1, \ldots, d$ one has the following:

1. For all $j^{\prime} \leq r$ with $j^{\prime} \neq j$, and for all $i=1, \ldots, q$, one has $\left(b_{j}\right)\left(i, j^{\prime}\right)=0$. Indeed, for this one need only exhibit a bad $S$ of Type 3 or 4 containing $\left(i, j^{\prime}\right)$ and $\left(k, j^{\prime}\right)$ and no element from column $j$. If, for instance, $n=8, d=3, r=2, k=q+1=3, j=$ $1, j^{\prime}=2, i=2$, then $S$ indicated by the stars in the following picture is such a bad $d$-set, of Type 3 :

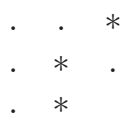

2. for all $j^{\prime}>r$ with $j^{\prime} \neq j$, and for all $i_{1}, i_{2}=1, \ldots, q$ with $i_{1} \neq i_{2}$ one has $\left(b_{j}\right)_{\left(i_{1}, j^{\prime}\right)}=\left(b_{j}\right)_{\left(i_{2}, j^{\prime}\right)}$. Indeed, for this one need only exhibit a bad $S$ of Type 3 or 4 containing $\left(i_{1}, j^{\prime}\right),\left(i_{2}, j^{\prime}\right)$ and no element from column $j$. If, for instance, $n=8, d=4, r=0, k=$ $q+1=3, j=2, j^{\prime}=3, i_{1}=1, i_{2}=2$, then

$$
\begin{array}{cccc}
\cdot & \cdot & * & * \\
* & \cdot & * & .
\end{array}
$$

(with an empty last row) is an example of such a bad $S$, of Type 4 .

This shows that the components of the $b_{j}$ that are not recorded by $B$ together contribute only a scalar multiple of $v_{k}$ to $w$. We may therefore just as well assume that those components are all 0 , i.e., that $b_{j}$ only has nonzero coefficients in positions in the $j$ th column of $A$. Hence now all coefficients of $e_{S}$ in $w$ are zero for $S$ of Type 3 or 4 .

Thus we end up looking for $B: A \rightarrow \mathbb{C}$ with the following properties:

1. For every bad $S$ of Type 1 , the sum $\sum_{(i, j) \in S} B(i, j)$ is 0 . Pictorially, the sum of $B$ along subsets of the form



is zero.

2. For every bad $S$ of Type 2 , the entry of $B$ at position $(i, j)$ (as in the definition of Type 2) is 0. Pictorially, if $S$ contains the positions of the stars in

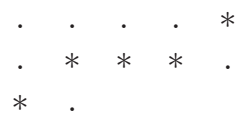

then $B(2,2)=0$.

3. For some good $S$ of Type 1 the sum above is nonzero, or for some good $S$ of Type 2 the entry $B(i, j)$ is nonzero - this to ensure that $w$ is a nonzero element of $T$. This condition will be expressed by calling $B$ nontrivial. 
For $(n, d, q+1) \in\{(8,4,3),(9,3,4)\}$ (where the $(q+1)$ st row of $A$ is empty) there certainly are such $B$ :

$$
\left[\begin{array}{cccc}
1 & 1 & 0 & 0 \\
0 & 0 & -1 & -1
\end{array}\right] \text { and }\left[\begin{array}{ccc}
1 & 1 & 1 \\
0 & 0 & 0 \\
-1 & -1 & -1
\end{array}\right] \text {. }
$$

Note that indeed, the sum along any subset $S$ of the indices that hits all columns is zero for bad $S$, while it can be nonzero if $S$ is good. For $(n, d, q+1)=(7,3,3)$ we can take the $B$ for $(n, d, q+1)=(9,3,4)$ and delete the (empty last row and) positions $(3,2)$ and $(3,4)$. This proves defectiveness of the Grassmannians in the proposition. (And with a little extra work, one shows that all $B$ with the required properties lead to scalar multiples of the same vector in $T_{v_{k}} C \cap T$, so that the defect is 1.)

Finally, we have to show that such $B$ exist only for the parameters above. First we argue that $r$ is at most 1 . Indeed, suppose that $r \geq 2$. Since $k-1=q \geq 2$, every choice of a position $(i, j)$ in $\{1, \ldots, k-1\} \times\{1, \ldots, r\}$, together with all entries $\left(k, j^{\prime}\right)$ with $j^{\prime} \leq r$ and $j^{\prime} \neq j$, can be extended to a bad $S$, having, in fact, positions in three different rows. This implies that $B(i, j)=0$ for all $i \leq k-1$ and $j \leq r$. Furthermore, all $S$ of Type 1 are bad (since $r \geq 2$ ), so that $B$ cannot be nontrivial.

So $r \leq 1$. First we handle $r=0$. We distinguish three cases:

1. If $d \geq 5$, then one readily sees that $B$ must be constant on each column of $A$ : For instance, for $(n, d)=(10,5)$ this follows for the third column of $A$ from the fact that the sum of $B$ is zero along both of the following bad $d$-sets:

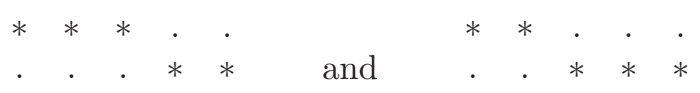

Now if $B$ is constant on each column, then its sums along all $S$ of Type 1 are identical; hence if that sum is zero for bad $S$, then it is also zero for all good $S$, but then $B$ cannot be nontrivial. This settles $d \geq 5$.

2. If $d=4$ and $q \geq 3$, then again $B$ is necessarily constant on every column of $A$; for instance, the fact that the sum of $B$ is zero along the two bad sets



shows that $B(2,4)=B(3,4)$. As in the case in which $d \geq 5$, this implies that $B$ cannot be nontrivial.
3. Finally, if $d=3$ and $n \geq 12$, then a similar reasoning using bad sets such as

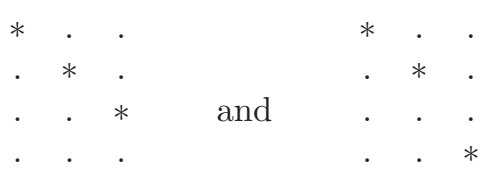

shows that again $B$ is constant on each column, hence trivial.

Next take $r=1$. Now we distinguish two cases:

1. $d \geq 4$. Then using bad $d$-sets of Type 2 such as

one finds that $B(i, 1)=0$ for all $i<k$. Also, the fact that $B$ sums up to 0 along $d$-sets of Type 1 such as

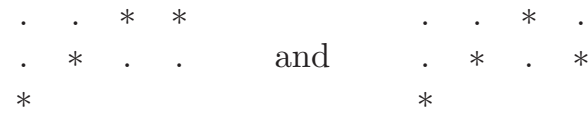

shows that $B$ is constant on each of the $d-1$ last columns of $A$. As before, this implies that $B$ does not meet the nontriviality condition.

2. $d=3$ and $n \geq 10$. Then bad $d$-sets of Type 2 such as

show that $B(i, 1)=0$ for all $i<k$. And finally, pairs of $d$-sets such as

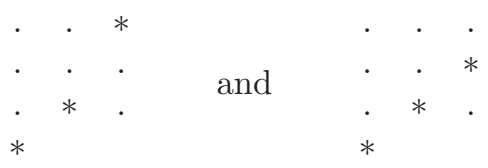

show that $B$ is constant on each of the $d-1$ last columns of $A$, hence not nontrivial.

This proves the proposition.

We cannot resist pointing out an interesting link between secant dimensions of Grassmannians and coding theory: a binary code of length $n$ and constant weight $d$ is a subset of $\{0,1\}^{n}$ in which every element has exactly $d$ entries equal to 1 . The (Hamming) distance between two 
elements of $\{0,1\}^{n}$ is the number of coordinates where they differ.

Theorem 4.3. Retain the setting of Conjecture 4.1, and let $B$ be a binary code of length $n$ and constant weight $d$ with $|B|=k$. Then the following hold:

1. If the distance between any two distinct elements of $B$ is at least 6 , then $k C$ is not defective.

2. If every word in $\{0,1\}^{n}$ of weight $d$ has an element of $B$ at distance at most 2 , then $k C=V$.

The first observation slightly generalizes [Catalisano et al. 05a, Theorem 2.1.ii]; the second observation is also stated, in a slightly different form, in [Ehrenborg 00, Proposition 7.5]. More relations between secant varieties and combinatorics are given in [Sturmfels and Sullivant 06].

Proof: Let $e_{1}, \ldots, e_{n}$ be a basis of $\mathbb{C}^{n}$. To every word $w$ in $\{0,1\}^{n}$ of weight $d$ we associate an element of $V$ as follows: If $i_{1}<\cdots<i_{d}$ are the coordinates $i$ where $b_{i}=1$, then we set

$$
e_{w}:=e_{i_{1}} \cdots e_{i_{d}} \in V
$$

Now

$$
T_{e_{w}} C=\sum_{j=1}^{d} e_{i_{1}} \cdots e_{i_{j-1}} \mathbb{C}^{n} e_{i_{j+1}} \cdots e_{i_{d}}
$$

is precisely the span of all $e_{u}$ where $u$ is a word of weight $d$ at distance at most 2 from $w$. Hence the dimension of $\sum_{b \in B} T_{e_{b}} C$ (and therefore that of $k C$ ) is at least the cardinality of the set of all weight- $d$ words in $\{0,1\}^{n}$ at distance at most 2 from $B$. This implies both statements.

\subsection{Segre powers}

For $G=\mathrm{SL}_{n}^{d}$ and $V=\left(\mathbb{C}^{n}\right)^{\otimes d}$, the minimal orbit $X$ is $\left(\mathbb{P}^{n-1}\right)^{d}$ in its Segre embedding, and the affine cone $C$ over $X$ is the set of decomposable tensors in $V$. For $d=2$ the set $k C$ corresponds to the set of $n \times n$ matrices of rank at most $k$, so we leave out this well-understood case from our study. Already for $d=3$, or for $d$ large and $n=2$, the secant dimensions are not known, though there are some results, for a good overview of which we refer to [Catalisano et al. 02]. Our conjecture is as follows.
Conjecture 4.4. Suppose that $d \neq 2$. The variety $C$ is defective if and only if

1. $n=2$ and $d=4$, in which case $(\operatorname{dim} k C)_{k}$ equals $(5,10, * 14,16)$; or

2. $n=3$ and $d=3$, in which case $(\operatorname{dim} k C)_{k}$ equals $(7,14,21, * 26,27)$,

where $*$ indicates the defective secant dimensions.

We have verified this conjecture with our program for $d+n \leq 8$ as well as for $n=2$ and $d=9,10$ and for $d=3$ and $n \leq 9$. Again, it is not hard but tedious to prove the following proposition.

Proposition 4.5. In the setting of Conjecture 4.4, $\mathrm{kC}$ is not defective for $k \leq n$, and $(n+1) C$ is defective if and only if $(n, d) \in\{(2,4),(3,3)\}$.

The proof is completely analogous to that of Proposition 4.2: Here $\left(\mathrm{SL}_{n}\right)^{d}$ has a dense orbit on $X^{k}$, whence the rank of the differential of the summation map $C^{k} \rightarrow$ $k C$ need be computed only at a point of $C^{k}$ over this orbit. We omit the details, but do report a funny numeric coincidence: The same array $B$ that shows that $3 C$ is defective for the Grassmannian of 4-dimensional subspaces of an 8-dimensional space also shows that $3 C$ is defective for the fourth Segre power of $\mathbb{P} \mathbb{C}^{2}$. The same (numeric) connection exists between the defect in $4 C$ for the Grassmannian of 3-dimensional subspaces of a 9-dimensional space and the defect in $4 C$ for the third Segre power of $\mathbb{P} \mathbb{C}^{3}$.

We recall another interesting link to coding theory, which can be proved in the same manner as Theorem 4.3, but is also well known from [Catalisano et al. 02, Ehrenborg 00, Sturmfels and Sullivant 06].

Theorem 4.6. In the setting of Conjecture 4.4, let $B$ be a subset of $\{1, \ldots, n\}^{d}$ of size $k$. Then the following hold:

1. If the Hamming distance between any two elements of $B$ is at least 3 , then $k C$ is not defective.

2. If every element of $\{1, \ldots, n\}^{d}$ is at distance at most 1 from an element of $B$, then $k C=V$.

\subsection{A Finiteness Question}

The experiments with our program suggest the following question: Fix the complex semisimple group $G$. To what extent is it true that the set of all irreducible representations of $G$ whose minimal orbit is defective is finite? For 
instance, from the fundamental work of Alexander and Hirschowitz [Alexander 88, Alexander and Hirschowitz 92, Alexander and Hirschowitz 95], we know that for each $n$, only finitely many symmetric powers of the natural representation of $\mathrm{SL}_{n}$ have defective minimal orbits.

We give a conjecturally complete list of "defective highest weights" for some small groups; note that for $G=\mathrm{SL}_{2}$, the minimal orbit is defective in no irreducible representation.

Conjecture 4.7. The only irreducible representations of $G$ for which the minimal orbit is defective are those with the following highest weights (in the labeling of [Bourbaki 687):

1. for $G$ of Type $A_{2}$ :

(a) $2 \omega_{1}$ and $2 \omega_{2}$ with secant dimensions $(3, * 5,6)$ (X is the quadratic Veronese embedding of $\left.\mathbb{P}^{2}\right)$,

(b) $4 \omega_{1}$ and $4 \omega_{2}$ with secant dimensions $(3,6,9,12, * 14,15)$ (the quartic Veronese embedding of $\left.\mathbb{P}^{2}\right)$,

(c) $\omega_{1}+\omega_{2}$ with secant dimensions $(4, * 7,8)$ (a hyperplane section of the Segre embedding of $\left.\mathbb{P}^{2} \times \mathbb{P}^{2}\right)$, and

(d) $2 \omega_{1}+2 \omega_{2}$ with secant dimensions $(4,8,12$, $16,20,24, * 26,27$ ) (the image of the previous variety under the quadratic Veronese reembedding of $\mathbb{P}\left(V_{\omega_{1}+\omega_{2}}\right)$, which image spans only a subspace of projective dimension 26 in the space $\mathbb{P} S^{2}\left(V_{\omega_{1}+\omega_{2}}\right)$ of dimension 35);

2. for $G$ of Type $A_{3}$ :

(a) $\omega_{1}+\omega_{2}$ and $\omega_{2}+\omega_{3}$ with secant dimensions $(6,12, * 17,20)$ (the flag variety of flags $V_{1} \subseteq V_{2} \subseteq$ $\mathbb{C}^{4}$ with $\operatorname{dim} V_{i}=i$, in its minimal-dimensional embedding),

(b) $2 \omega_{1}$ and $2 \omega_{3}$ with secant dimensions $(4, * 7, * 9,10)$ (the quadratic Veronese embedding of $\mathbb{P}^{3}$ ),

(c) $\omega_{1}+\omega_{3}$ with secant dimensions $(6, * 11, * 14,15)$ (a hyperplane section of the Segre embedding of $\left.\mathbb{P}^{3} \times \mathbb{P}^{3}\right)$,

(d) $2 \omega_{2}$ with secant dimensions $(5,10, * 14$, $* 17, * 19,20) \quad$ (the image of the Grassmannian of 2-spaces in 4-space under the quadratic Veronese reembedding of $\mathbb{P} V_{\omega_{2}}$; this image spans a codimension-1 subspace), and

(e) $4 \omega_{1}$ and $4 \omega_{3}$ with secant dimensions $(4,8,12,16,20,24,28,32, * 34,35) \quad$ (the quartic Veronese embedding of $\mathbb{P}^{3}$ );
3. for $G$ of Type $B_{2}$ :

(a) $2 \omega_{1}$ with secant dimensions $(4,8, * 11, * 13,14)$ (the image of a quadric in $\mathbb{P}^{4}$ under the quadratic Veronese reembedding, which image spans a codimension-1 subspace),

(b) $2 \omega_{2}$ with secant dimensions $(4, * 7, * 9,10)$ (the image of the Grassmannian of isotropic 2dimensional vector spaces under the quadratic Veronese reembedding),

(c) $\omega_{1}+\omega_{2}$ with secant dimensions $(5,10, * 14,16)$ (the flag variety of isotropic flags $V_{1} \subseteq V_{2} \subseteq \mathbb{C}^{5}$ with $\operatorname{dim} V_{i}=i$, in its minimal embedding), and

(d) $4 \omega_{2}$ with secant dimensions $(4,8, \ldots$, $28,32, * 34,35$ ) (the quartic Veronese reembedding of the Grassmannian of isotropic 2-dimensional vector spaces in $\mathbb{C}^{4}$ ); and, finally,

4. for $G$ of Type $G_{2}$ :

(a) $2 \omega_{1}$ with secant dimensions $(6,12, * 17, * 21,24)$ (the quadratic Veronese reembedding of a quadric in $\mathbb{P}^{6}$, spanning a codimension-1 subspace),

(b) $\omega_{2}$ with secant dimensions $(6, * 11,14)$ (the orbit of long-root vectors in $\left.G_{2}\right)$, and

(c) $2 \omega_{2}$ with secant dimensions $(6, \ldots, 72, * 76,77)$ (the quadratic Veronese reembedding of the previous variety, which image spans a proper subspace).

The conjecture for $A_{2}$ and $B_{2}$ has been verified for weights $i \omega_{1}+j \omega_{2}$ with $i+j \leq 6$. For $A_{3}$, the conjecture has been checked for highest weights $i \omega_{1}+j \omega_{2}+k \omega_{3}$ with $i+j+k \leq 4$, and for $G_{2}$ the conjecture has been verified for all highest weights $i \omega_{1}+j \omega_{2}$ with $i+j \leq 4$. To attack the question posed in this section, one would need completely new techniques, far beyond our easy algorithm. But we hope that the challenges boldly posed in this paper as conjectures will be taken up by some of our readers!

\section{ACKNOWLEDGMENTS}

The first author was supported by EPSRC grant number GR/S35387/01. The second author was supported by NWO mathematics cluster DIAMANT. 


\section{REFERENCES}

[Alexander 88] J. Alexander. "Singularités imposables en position générale à une hypersurface projective." Compos. Math. 68:3 (1988), 305-354.

[Alexander and Hirschowitz 92] J. Alexander and A. Hirschowitz. "La méthode d'Horace éclatée: Application à l'interpolation en degré quatre." Invent. Math. 107:3 (1992), 585-602.

[Alexander and Hirschowitz 95] J. Alexander and A. Hirschowitz. "Polynomial Interpolation in Several Variables." J. Algebr. Geom. 4:2 (1995), 201-222.

[Baur and Draisma 04] Karin Baur and Jan Draisma. "Higher Secant Varieties of the Minimal Adjoint Orbit." J. Algebra 280 (2004), 743-761.

[Borel 91] Armand Borel. Linear Algebraic Groups. New York: Springer-Verlag, 1991.

[Bourbaki 68] Nicolas Bourbaki. Groupes et Algèbres de Lie (Chapters IV, V, VI), Éléments de mathématique XXXIV. Paris: Hermann, 1968.

[Bruns and Vetter 88] Winfried Bruns and Udo Vetter. Determinantal Rings, Lecture Notes in Mathematics, 1327. New York: Springer-Verlag, 1988.

[Catalisano et al. 02] M. V. Catalisano, A. V. Geramita, and A. Gimigliano. "On the Rank of Tensors, via Secant Varieties and Fat Points." In Zero-Dimensional Schemes and Applications. Proceedings of the Workshop, Naples, Italy, February 9-12, 2000, edited by A. V. Geramita, pp. 135-147, Queen's Pap. Pure Appl. Math. 123. Kingston: Queen's University, 2002.

[Catalisano et al. 05a] M. V. Catalisano, A. V. Geramita, and A. Gimigliano. "Secant Varieties of Grassmann Varieties." Proc. Am. Math. Soc. 133:3 (2005), 633-642.

[Catalisano et al. 05b] M. V. Catalisano, A. V. Geramita, and A. Gimigliano. "Higher Secant Varieties of the Segre Varieties $\mathbb{P}^{1} \times \cdots \times \mathbb{P}^{1}$." J. Pure Appl. Algebra 201:1-3 (2005), 367-380.

[Ehrenborg 00] R. Ehrenborg. "On Apolarity and Generic Canonical Forms." J. of Algebra 213 (1999), 167-194.

[GAP 02] The GAP Group. GAP-Groups, Algorithms, and Programming, Version 4.3. Available online (http://www. gap-system.org), 2002.

[de Graaf 03] W. A. de Graaf. "An Algorithm to Compute the Canonical Basis of an Irreducible Module over a Quantized Enveloping Algebra." LMS J. Comput. Math. 6 (2003), 105-118.

[de Graaf 05] W. A. de Graaf. "QuaGroup." A GAP package, version 1.1 (see [GAP 02]), 2005.
[Harris 92] Joe Harris. Algebraic Geometry. A First Course. Graduate Texts in Mathematics 133. New York: SpringerVerlag, 1992.

[Hirschowitz 85] André Hirschowitz. "La méthode d'Horace pour l'interpolation à plusieurs variables." Manuscr. Math. 50 (1985), 337-388.

[Humphreys 71] J. E. Humphreys. Introduction to Lie Algebras and Representation Theory. New York: SpringerVerlag, 1972.

[Humphreys 81] James E. Humphreys. Linear Algebraic Groups, Graduate Texts in Mathematics 21. New York: Springer-Verlag, 1981.

[Iarrobino and Kanev 99] Anthony Iarrobino and Vassil Kanev. Power sums, Gorenstein Algebras, and Determinantal Loci, Lecture Notes in Mathematics 1721. New York: Springer-Verlag, 1999.

[Jantzen 96] J. C. Jantzen. Lectures on Quantum Groups, Graduate Studies in Mathematics 6. Providence: American Mathematical Society, 1996.

[Kaji and Yasukura 00] Hajime Kaji and Osami Yasukura. "Secant Varieties of Adjoint Varieties: Orbit Decomposition." J. Algebra 227:1 (2000), 26-44.

[Kaji et al. 99] Hajime Kaji, Masahiro Ohno, and Osami Yasukura. "Adjoint Varieties and Their Secant Varieties." Indag. Math., New Ser. 10:1 (1999), 45-57.

[Lakshmibai 95] V. Lakshmibai. "Bases for Quantum Demazure Modules." In Representations of Broups (Banff, AB, 1994), pp. 199-216. Providence: Amer. Math. Soc., 1995.

[Landsberg and Weyman 06] J. M. Landsberg and Jerzy Weyman. "On the Ideals and Sof Secant Varieties of Segre Varieties." Preprint, available online (http://www.arxiv. org/pdf/math.AG/0601452), 2006.

[McGillivray 05] Barbara McGillivray. "A Probabilistic Algorithm for the Secant Defect of Grassmann Varieties." Preprint, available online (http://arxiv.org/abs/math. AG/0511683), 2005.

[Steinberg 68] Robert Steinberg. Lectures on Chevalley Groups. New Haven: Yale University Press, 1968.

[Sturmfels and Sullivant 06] Bernd Sturmfels and Seth Sullivant. "Combinatorial Secant Varieties." J. Pure Applied Math. Quarterly 2:3 (2006), 867-891.

[Terracini 11] A. Terracini. "Sulle $V_{k}$ per cui la varietà degli $S_{h}(h+1)$-seganti ha dimensione minore dell' ordinario. Palermo Rend. 31 (1911), 392-396.

[Zak 93] F. L. Zak. Tangents and Secants of Algebraic Varieties, Translations of Mathematical Monographs 127. Providence: American Mathematical Society, 1993. 
Karin Baur, Department of Mathematics, University of Leicester, University Road, Leicester LE1 7RH, U.K. (k.baur@mcs.le.ac.uk)

Jan Draisma, Department of Mathematics and Computer Science, Technische Universiteit Eindhoven, P.O. Box 513, 5600 MB Eindhoven, Netherlands (j.draisma@tue.nl)

Willem A. de Graaf, Dipartimento di Matematica, Universitá degli studi di Trento, Via Sommarive 14, I-38050 Povo (Trento), Italy (degraaf@science.unitn.it)

Received April 25, 2006; accepted in revised form October 11, 2006. 